SELECTION OF ANESTHESIA

By John Adriani, M.D. Pp. xvii +327 , illustrated. Oxford: Blackwell Scientific Publications. I 955. $47 \mathrm{~s} .6 \mathrm{~d}$.

This book contains the elementary principles of anaesthesia, set out in a delightfully brief and simple manner. It is intended largely for those American surgeons who are responsible for prescribing anaesthetic methods to nurse anaesthetists, but can be wholly recommended to beginners in the subject in this country.

It is divided into three parts. The first contains the pharmacology and clinical application of drugs used by the anaesthetist, the pharmacological data being sufficient for practical purposes only. Not all the newer drugs are included, chlorpromazine being a notable omission. The section ends with a chapter on anaesthetic deaths.

The second part describes possible medical complications in surgical patients and the manner in which various anaesthetics may affect them.

The last section considers the choice of anaesthetic in relation to the operation to be performed.

Adriani shares to some extent his fellow countryman Beecher's timidity towards relaxants and recommends spinal analgesia more frequently than would many/British anaesthetists.

\section{PREMATURE INFANTS}

\section{A Manual for Physicians}

By Ethel C. Dunham, M.D. Second Edition. Pp. xii +459 , with $4 \mathrm{r}$ illustrations. London: Cassell \& Co., Ltd. I955. 63s.

The original edition of this book was a papercovered publication of the Children's Bureau of the United States of America and cost \$1.25. This second edition is a much more elegant production and contains an even greater wealth of information. A person organising a premature unit in any country in the world would be well advised to study this book closely. He will then learn how people have overcome some of the problems in the care of the premature infant in different ways. He will not find that Dr. Dunham has made up his mind for him. She realizes too well that there is not necessarily only one correct solution to a problem: Oddly enough, the section on Care in the Neonatal Period is the least pleasing, as every reader would like to know what she herself thinks is best, for example, about the temperature in the incubator. for different sized babies.
Part I-General Considerations-and Part 3Public Health Considerations - are both outstanding.

\section{MODERN TRENDS IN ORTHOPAEDICS}

(Second Series)

Edited by Sir Harry Platt, LL.D., M.D., M.S., P.R.C.S., F.A.C.S. Pp. ix +33 , with 226 illustrations. London: Butterworth \& Co. Ltd. r $956.65 \mathrm{~s}$.

This new volume contains eleven essays on topics of contemporary interest in orthopaedic surgery. The choice of title is not particularly felicitous, for several of the essays are really summaries of previous work rather than indications of modern trends. Thus, Capener reviews the development of the reconstructive surgery of the hip joint, Barnes summarizes the results of wartime work on injuries of peripheral nerves, Grant Bonnin describes various conditions affecting the knee joint, and Gray reminds us of the difficulties of treating fractures of the femoral neck. These essays, valuable though they are for their clear presentation, are perhaps more suitable for a text book than for a book professing to show the way ahead.

However, the Editor himself gives us much to think about in his discussion on the treatment of congenital dislocation of the hip, while Holdsworth, in his treatise on traumatic paraplegia, and Seddon, in his review of Pott's paraplegia, make points which will be new to many and interesting to all. These three monographs are of outstanding interest and of permanent value. For the rest, Clark's contribution on muscle and tendon transference provides a useful description of these reconstructive procedures, and Pulvertaft provides an equally useful guide to treatment of contractures of the hand. Dobson deals with antibiotics in the treatment of bone and joint tubercle, while Scott contributes a clear presentation of the difficult subject of coxa vara.

The production of the book is good and the standard of illustration generally is high, though several of the plates (figs. I I 8, I 39, I4I, I65a) have been printed upside-down and it is distressing to find in the Table of Contents that the Oxford Hospitals are "Unilateral." It is, however, easy to disregard these small faults in a book which will be of interest and of value to all concerned in the development of orthopaedic surgery.

' G.B.'

BACK NUMBERS OF THE POSTGRADUATE MEDICAL JOURNAL

If any subscribers have copies, in good condition, of MARCH and APRIL 1952, the Fellowship of Postgraduate Medicine, 60 Portland Place, London, W.I, will be glad to purchase them. 